\title{
Deep learning assisted optical wavefront shaping in disordered medium (Erratum)
}

, "Deep learning assisted optical wavefront shaping in disordered medium (Erratum)," Proc. SPIE 10886, Adaptive Optics and Wavefront Control for Biological Systems V, 108861J (17 April 2019); doi: 10.1117/12.2535809

SPIE. Event: SPIE BiOS, 2019, San Francisco, California, United States 


\title{
Deep learning assisted optical wavefront shaping in disordered medium (Erratum)
}

\author{
Yunqi Luo*a, Huanhao Li ${ }^{\text {b }}$, Ruochong Zhang ${ }^{\text {a }}$, Puxiang Lai ${ }^{\mathrm{b}}$, Yuanjin Zheng ${ }^{\mathrm{a}}$ \\ ${ }^{a}$ Nanyang Technological University, 50 Nanyang Avenue,Singapore 639798; ${ }^{b}$ Hong Kong \\ Polytechnic University, 11 Yuk Choi Rd, Hung Hom, Hong Kong, China
}

\section{A revised version of}

Yunqi Luo, Huanhao Li, Ruochong Zhang, Puxiang Lai, and Yuanjin Zheng "Deep learning assisted optical wavefront shaping in disordered medium", Proc. SPIE 10886, Adaptive Optics and Wavefront Control for Biological Systems V, 1088612 (20 February 2019); doi:

10.1117/12.2504425 was published on 17 April 2019.

Details of the revision are provided in the text that accompanies this Erratum. The original paper has been updated.

A citation to 'Light scattering control in transmission and reflection with neural networks' (Optics Express Vol. 26, Issue 23, pp. 30911-30929, 2018), as reference [11], has been added in Section 2, paragraph 4. Figure 1 and its caption have been modified as follows:

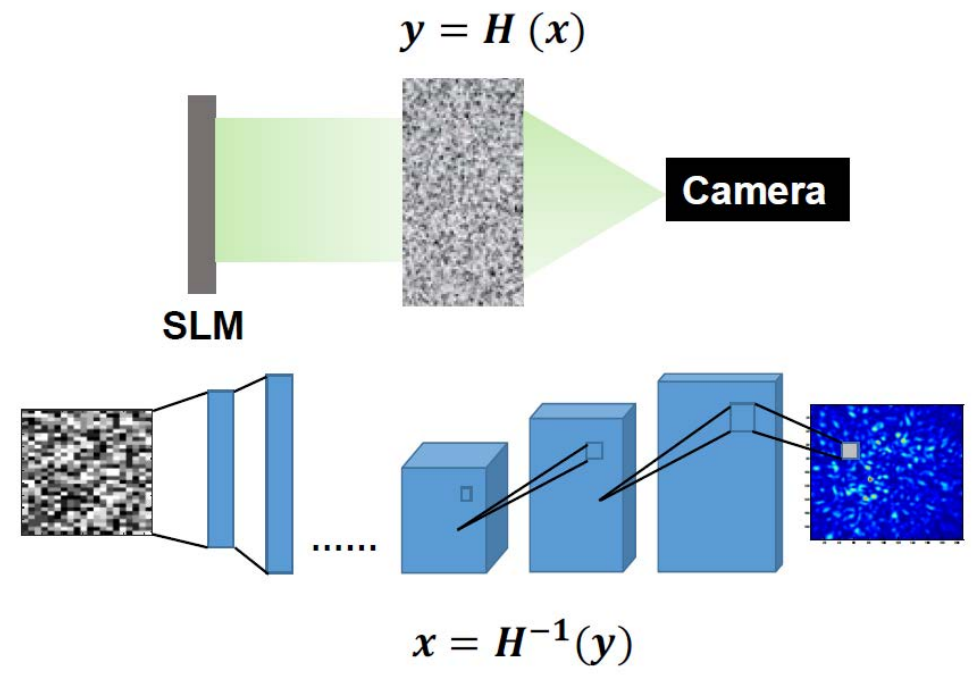

Figure 1. Illustration of wavefront shaping using neural networks. Light undergoes multiple scattering inside inhomogeneous media, and incident light with different SLM patterns results in different output speckle patterns. The speckle patterns serve as inputs to a pre-constructed convolutional neural network while the SLM patterns as the outputs. After proper training, convolutional neural networks are able to establish the relationship between the speckle patterns to their corresponding SLM patterns.

The authors would like to apologize for the inconvenience caused for readers and state that these do not change the scientific conclusions of the article in any way. The original article has been correspondingly updated.

* Correspondence: luoy0025@e.ntu.edu.sg;

Adaptive Optics and Wavefront Control for Biological'Systems V, edited by Thomas G. Bifano, Sylvain Gigan, Na Ji, Proc. of SPIE Vol. 10886, 108861J · @ 2019 SPIE · CCC code: 1605-7422/19/\$18 · doi: 10.1117/12.2535809 